Pacific

Journal of

Mathematics

\title{
HYPERBOLIC STRUCTURES ON CLOSED SPACELIKE MANIFOLDS
}

KUN ZHANG 


\title{
HYPERBOLIC STRUCTURES ON CLOSED SPACELIKE MANIFOLDS
}

\author{
KUN ZHANG
}

\begin{abstract}
We give an intrinsic generalization of spacelike manifolds. We define the intrinsic mean curvature flow and study it on certain closed generalized spacelike manifolds. Then we prove the existence of hyperbolic structures on them.
\end{abstract}

\section{Introduction}

Recall that a Riemannian manifold $(M, g)$ is hyperbolic if it has constant negative sectional curvature. These manifolds all come from the quotient of hyperbolic space $H^{n}$ by discrete isometry groups. However, it is difficult to find a good intrinsic characterization for whether hyperbolic structures exist on a given manifold. First, we know that some negatively pinched Riemannian manifolds do not admit a hyperbolic metric. The $n \geq 4$ counterexample in [Gromov and Thurston 1987] contrasts sharply with the pinching theorem of positively curved manifolds, and implies that is it not always possible to deform by geometric flow a given negatively curved metric into one with constant negative curvature. However, this paper will show that a hyperbolic structure exists naturally on a large class of manifolds.

Consider the well known model of hyperbolic space by the imaginary unit sphere in Minkowski space $\mathbb{R}^{1, n}$, where the Minkowski metric in Cartesian coordinates $\left(x^{0}, x^{1}, \ldots, x^{n}\right)$ is

$$
g=-\left(d x^{0}\right)^{2}+\left(d x^{1}\right)^{2}+\cdots+\left(d x^{n}\right)^{2}
$$

and the equation of the imaginary unit sphere is

$$
-\left(x^{0}\right)^{2}+\left(x^{1}\right)^{2}+\cdots+\left(x^{n}\right)^{2}=-1 .
$$

That the imaginary unit sphere has sectional curvature equal to -1 can be seen from the Gauss-Codazzi equations

$$
R_{i j k l}-\left(h_{i l} h_{j k}-h_{i k} h_{j l}\right)=0 \quad \text { and } \quad \nabla_{i} h_{j k}-\nabla_{j} h_{i k}=0,
$$

MSC2000: 53C44.

Keywords: hyperbolic structures, closed spacelike manifolds, mean curvature flow. 
where $h_{i j}$ is the second fundamental form, and $h_{i j}$ equals $g_{i j}$ on the imaginary unit sphere. In this paper, we are interested in an intrinsic generalization of this model.

Definition 1.1. We call a triple $\left(M, g_{i j}, h_{i j}\right)$ a spacelike manifold if $\left(M, g_{i j}\right)$ is a Riemannian manifold and $h_{i j}$ is a symmetric tensor satisfying the Gauss-Codazzi equations

$$
R_{i j k l}-\left(h_{i l} h_{j k}-h_{i k} h_{j l}\right)=0 \text { and } \quad \nabla_{i} h_{j k}-\nabla_{j} h_{i k}=0 .
$$

Remark 1.2. It follows from this definition that there is a locally isometric and spacelike embedding of $(M, g)$ into $\mathbb{R}^{1, n}$, and we can globally embed the universal cover of $(M, g)$ into $\mathbb{R}^{1, n}$ as a spacelike hypersurface.

Our main theorem is this:

Theorem 1.3. Let $(M, g, h)$ be an n-dimensional closed spacelike manifold with $h_{i j}>0$ and $n \geq 4$. Then $M$ admits a hyperbolic metric.

The idea is to use geometric flows. In contrast with extrinsic mean curvature flow, we define an intrinsic mean curvature flow of $(g, h)$ by

$$
\begin{aligned}
& \partial_{t} g_{i j}=-2 R_{i j}+2 h_{i m} h_{n j} g^{m n}, \\
& \partial_{t} h_{i j}=\triangle h_{i j}-R_{i m} h_{n j} g^{m n}-R_{j m} h_{n i} g^{m n}+2 h_{i k} h_{l m} h_{n j} g^{k l} g^{m n}-|A|^{2} h_{i j},
\end{aligned}
$$

with $g_{i j}(x, 0)=\tilde{g}_{i j}(x)$, the initial metric on $M$, and $h_{i j}(x, 0)=\tilde{h}_{i j}(x)$, the initial data of $h_{i j}$. Here $|A|^{2}=g^{i k} g^{j l} h_{i j} h_{k l}$ and $\partial_{t}:=\partial / \partial_{t}$. We will solve (1-1) intrinsically and show that the solution exists for all times in $[0, \infty)$ and converges (after normalization) to a hyperbolic metric.

Remark 1.4. Mean curvature flows have been intensively studied in recent years. See [Huisken 1984] for Euclidean ambient space and [Ecker and Huisken 1991; Ecker 1997] for Minkowski ambient space. Note that in extrinsic mean curvature flow (with ambient space $\mathbb{R}^{1, n}$ ), we deform the position vector $F$ by the evolution equation $\partial F / \partial t=-H$. With the Gauss-Codazzi equations we begin with the equations of the metric and the second fundamental form that come from the extrinsic mean curvature flow, and change them to the weakly parabolic system (1-1). This system is intrinsically defined and interesting in its own right.

Remark 1.5. In [Ecker 1997], it was shown that there is a long-time solution for mean curvature flow of noncompact spacelike hypersurfaces in Minkowski space. If we were to lift $(M, g)$ to its universal cover and deform the universal cover by the extrinsic mean curvature flow, we would get a long-time solution. Then to get an induced solution on $M$, we would need a uniqueness theorem for the mean curvature flow. Here, we try a completely different method. 


\section{Short-time existence and uniqueness}

System (1-1) is not strictly parabolic, so in order to apply the theory of such systems to get short-time existence, we will use a trick of De Turck: We will combine our evolution (1-1) with the harmonic map flow.

Let $\left(M^{n}, g_{i j}(x)\right)$ and $\left(N^{m}, s_{\alpha \beta}(y)\right)$ be Riemannian manifolds with $F: M^{n} \rightarrow N^{m}$ a map between them. The harmonic map flow is an evolution equation for maps from $M^{n}$ to $N^{m}$ and is given by

$$
\begin{aligned}
\partial_{t} F(x, t) & =\Delta F(x, t) & & \text { for } x \in M^{n} \text { and } t>0, \\
F(x, 0) & =F(x) & & \text { for } x \in M^{n},
\end{aligned}
$$

where $\triangle$ is defined by using the metrics $g_{i j}(x)$ and $s_{\alpha \beta}(y)$ through

$$
\triangle F^{\alpha}(x, t)=g^{i j}(x) \nabla_{i} \nabla_{j} F^{\alpha}(x, t),
$$

and

$$
\nabla_{i} \nabla_{j} F^{\alpha}(x, t)=\frac{\partial^{2} F^{\alpha}}{\partial x^{i} \partial x^{j}}-\Gamma_{i j}^{k} \frac{\partial F^{\alpha}}{\partial x^{k}}+\tilde{\Gamma}_{\beta \gamma}^{\alpha} \frac{\partial F^{\beta}}{\partial x^{i}} \frac{\partial F^{\gamma}}{\partial x^{j}} .
$$

Here we use $\left\{x^{i}\right\}$ and $\left\{y^{\alpha}\right\}$ to denote the local coordinates of $M^{n}$ and $N^{m}$, respectively, and $\Gamma_{i j}^{k}$ and $\tilde{\Gamma}_{\beta \gamma}^{\alpha}$ are the corresponding Christoffel symbols of $g_{i j}$ and $s_{\alpha \beta}$. The harmonic map flow is strictly parabolic, so for any initial data, there exists a short-time smooth solution.

Let $\left(g_{i j}(x, t), h_{i j}(x, t)\right)$ be a complete smooth solution of our system (1-1). Then the harmonic map flow coupled with our evolution equation is the system

$$
\begin{aligned}
\partial_{t} F(x, t) & =\triangle_{t} F(x, t) & & \text { for } x \in M^{n} \text { and } t>0, \\
F(x, 0) & =\text { identity } & & \text { for } x \in M^{n},
\end{aligned}
$$

where $\triangle_{t}$ is defined by using the metrics $g_{i j}(x, t)$ and $s_{\alpha \beta}(y)$.

Let $\left(F^{-1}\right)^{*} g$ and $\left(F^{-1}\right)^{*} h$ be the one-parameter families of pulled-back metrics and tensors on the target $\left(N^{n}, s_{\alpha \beta}\right)$. Write $\hat{g}_{\alpha \beta}(y, t)=\left(\left(F^{-1}\right)^{*} g\right)_{\alpha \beta}(y, t)$ and $\hat{h}_{\alpha \beta}(y, t)=\left(\left(F^{-1}\right)^{*} h\right)_{\alpha \beta}(y, t)$. Then by direct calculations, $\hat{g}_{\alpha \beta}(y, t)$ and $\hat{h}_{\alpha \beta}(y, t)$ satisfy the evolution equations

$$
\begin{aligned}
\partial_{t} \hat{g}_{\alpha \beta}(y, t)= & -2 \hat{R}_{\alpha \beta}(y, t)+2 \hat{h}_{\alpha \sigma} \hat{h}_{\rho \beta} \hat{g}^{\sigma \rho}+\nabla_{\alpha} V_{\beta}+\nabla_{\beta} V_{\alpha} \\
\partial_{t} \hat{h}_{\alpha \beta}(y, t)= & \triangle \hat{h}_{\alpha \beta}(y, t)-\hat{R}_{\alpha \sigma} \hat{h}_{\rho \beta} \hat{g}^{\sigma \rho}-\hat{R}_{\beta \sigma} \hat{h}_{\rho \alpha} \hat{g}^{\sigma \rho} \\
& +2 \hat{h}_{\alpha \lambda} \hat{h}_{\mu \nu} \hat{h}_{\rho \beta} \hat{g}^{\lambda \mu} \hat{g}^{v \rho}-|\hat{A}|^{2} \hat{h}_{\alpha \beta}+\hat{h}_{\beta \gamma} \nabla_{\alpha} V^{\gamma}+\hat{h}_{\alpha \gamma} \nabla_{\beta} V^{\gamma},
\end{aligned}
$$

where $V^{\alpha}=g^{\beta \gamma}\left(\Gamma_{\beta \gamma}^{\alpha}(\hat{g})-\tilde{\Gamma}_{\beta \gamma}^{\alpha}(s)\right)$, and $\Gamma_{\beta \gamma}^{\alpha}(\hat{g})$ and $\tilde{\Gamma}_{\beta \gamma}^{\alpha}(s)$ are the Christoffel symbols of the metrics $\hat{g}_{\alpha \beta}(y, t)$ and $s_{\alpha \beta}(y)$, respectively. Here we analyze the 
principal part of the right side of (2-4). One can see that $-2 \hat{R}_{\alpha \beta}(y, t)+2 \hat{h}_{\alpha \sigma} \hat{h}_{\rho \beta} \hat{g}^{\sigma \rho}+\nabla_{\alpha} V_{\beta}+\nabla_{\beta} V_{\alpha}=\hat{g}^{\mu \nu} \frac{\partial^{2} \hat{g}_{\alpha \beta}}{\partial y^{\mu} \partial y^{\nu}}+$ (lower order terms) and

$$
\begin{aligned}
\Delta \hat{h}_{\alpha \beta}(y, t)-\hat{R}_{\alpha \sigma} \hat{h}_{\rho \beta} \hat{g}^{\sigma \rho}-\hat{R}_{\beta \sigma} \hat{h}_{\rho \alpha} \hat{g}^{\sigma \rho} & \\
+ & 2 \hat{h}_{\alpha \lambda} \hat{h}_{\mu \nu} \hat{h}_{\rho \beta} \hat{g}^{\lambda \mu} \hat{g}^{\nu \rho}-|\hat{A}|^{2} \hat{h}_{\alpha \beta}+\hat{h}_{\beta \gamma} \nabla_{\alpha} V^{\gamma}+\hat{h}_{\alpha \gamma} \nabla_{\beta} V^{\gamma} \\
= & \hat{g}^{\mu \nu}\left(\frac{\partial^{2} \hat{h}_{\alpha \beta}}{\partial y^{\mu} \partial y^{\nu}}-\frac{\partial \Gamma_{\alpha \mu}^{\sigma}}{\partial y^{\nu}} \hat{h}_{\sigma \beta}-\frac{\partial \Gamma_{\beta \mu}^{\sigma}}{\partial y^{\nu}} \hat{h}_{\sigma \alpha}\right)-\hat{g}^{\mu \nu}\left(-\frac{\partial \Gamma_{\alpha \mu}^{\sigma}}{\partial y^{\nu}}+\frac{\partial \Gamma_{\mu \nu}^{\sigma}}{\partial y^{\alpha}}\right) \hat{h}_{\sigma \beta} \\
& -\hat{g}^{\mu \nu}\left(-\frac{\partial \Gamma_{\beta \mu}^{\sigma}}{\partial y^{\nu}}+\frac{\partial \Gamma_{\mu \nu}^{\sigma}}{\partial y^{\beta}}\right) \hat{h}_{\sigma \alpha}+\hat{g}^{\mu \nu} \frac{\partial \Gamma_{\mu \nu}^{\gamma}}{\partial y^{\alpha}} \hat{h}_{\gamma \beta}+\hat{g}^{\mu \nu} \frac{\partial \Gamma_{\mu \nu}^{\gamma}}{\partial y^{\beta}} \hat{h}_{\gamma \alpha} \\
& + \text { (lower order terms) } \\
=\hat{g}^{\mu \nu} \frac{\partial^{2} \hat{h}_{\alpha \beta}}{\partial y^{\mu} \partial y^{\nu}}+\text { (lower order terms). } &
\end{aligned}
$$

Hence

$$
\begin{aligned}
& \partial_{t} \hat{g}_{\alpha \beta}(y, t)=\hat{g}^{\mu \nu} \frac{\partial^{2} \hat{g}_{\alpha \beta}}{\partial y^{\mu} \partial y^{\nu}}+\text { (lower order terms), } \\
& \partial_{t} \hat{h}_{\alpha \beta}(y, t)=\hat{g}^{\mu \nu} \frac{\partial^{2} \hat{h}_{\alpha \beta}}{\partial y^{\mu} \partial y^{\nu}}+\text { (lower order terms), }
\end{aligned}
$$

and we know (2-4) is a strictly parabolic system. By the theory of such equations, there exists a smooth short-time solution of (2-4) for any initial data.

We can recover the solution $(g, h)$ for the original evolution equations from the solution $(\hat{g}, \hat{h})$, as follows. Let $\left(N^{n}, s_{\alpha \beta}\right)=\left(M^{n}, g_{\alpha \beta}(\cdot, 0)\right)$. Since

$$
V^{\alpha}=g^{\beta \gamma}\left(\Gamma_{\beta \gamma}^{\alpha}(\hat{g})-\tilde{\Gamma}_{\beta \gamma}^{\alpha}(s)\right)=-\left(\triangle F \circ F^{-1}\right)^{\alpha},
$$

we have

$$
\partial_{t} F=-V \circ F \text {. }
$$

Now once we have $\hat{g}_{\alpha \beta}$, we know $V$ and can solve (2-7), which is just a system of ordinary differential equations on the domain $M$. Hence $(g, h)$ can be recovered as the pull-backs $g=F^{*} \hat{g}$ and $h=F^{*} \hat{h}$.

Now we claim uniqueness of the solutions of (1-1) with given smooth initial conditions on a compact manifold. Suppose $\left(g_{1}, h_{1}\right)$ and $\left(g_{2}, h_{2}\right)$ are two solutions that agree at $t=0$. We can solve the coupled harmonic map flow (2-3) for maps $F_{1}$ and $F_{2}$ with the metrics $g_{1}$ and $g_{2}$ on $M$ into the same target $N$, with the same fixed $s$ and initial data. Then we have two solutions $\hat{g}_{1}$ and $\hat{g}_{2}$ on $N$ with the same initial metric. By the standard uniqueness result for strictly parabolic equations, we 
have $\left(\hat{g}_{1}, \hat{h}_{1}\right)=\left(\hat{g}_{2}, \hat{h}_{2}\right)$. Hence by (2-6) the corresponding vector fields $V_{1}=V_{2}$. Then the solutions of the ordinary differential equations $\partial_{t} F_{1}=-V_{1} \circ F_{1}$ and $\partial_{t} F_{2}=-V_{2} \circ F_{2}$ with the same initial values must coincide, and the solutions $\left(g_{1}, h_{1}\right)=F^{*}\left(\hat{g}_{1}, \hat{h}_{1}\right)$ and $\left(g_{2}, h_{2}\right)=F^{*}\left(\hat{g}_{2}, \hat{h}_{2}\right)$ of $(1-1)$ must agree.

\section{Preservation of the Gauss-Codazzi equations}

Here we will show that the Gauss-Codazzi equations are preserved under (1-1). Let $G_{i j k l}=R_{i j k l}-\left(h_{i l} h_{j k}-h_{i k} h_{j l}\right)$ and $C_{i j k}=\nabla_{i} h_{j k}-\nabla_{j} h_{i k}$.

Proposition 3.1. If the tensor $h_{i j}$ satisfies the Gauss-Codazzi equations

$$
R_{i j k l}-\left(h_{i l} h_{j k}-h_{i k} h_{j l}\right)=0 \quad \text { and } \quad \nabla_{i} h_{j k}-\nabla_{j} h_{i k}=0
$$

at time $t=0$, then it also does so for $t>0$.

Proof. By direct calculations, we have

$$
\begin{aligned}
\partial_{t} \Gamma_{i j}^{k} & =\frac{1}{2} g^{k l}\left(\nabla_{j}\left(\partial_{t} g_{i l}\right)+\nabla_{i}\left(\partial_{t} g_{j l}\right)-\nabla_{l}\left(\partial_{t} g_{i j}\right)\right), \\
\partial_{t} R_{i j l}^{k} & =\nabla_{i}\left(\partial_{t} \Gamma_{j l}^{k}\right)-\nabla_{j}\left(\partial_{t} \Gamma_{i l}^{k}\right), \\
\partial_{t} R_{i j k l} & =g_{h k} \partial_{t} R_{i j l}^{h}+\partial_{t} g_{h k} R_{i j l}^{h} .
\end{aligned}
$$

With these identities we get

$$
\begin{aligned}
\partial_{t} R_{i j k l}=\nabla_{i} & \nabla_{k} R_{j l}-\nabla_{i} \nabla_{l} R_{j k}-\nabla_{j} \nabla_{k} R_{i l}+\nabla_{j} \nabla_{l} R_{i k} \\
& -\nabla_{i} \nabla_{k}\left(h_{j m} h_{n l} g^{m n}\right)+\nabla_{i} \nabla_{l}\left(h_{j m} h_{n k} g^{m n}\right) \\
& +\nabla_{j} \nabla_{k}\left(h_{i m} h_{n l} g^{m n}\right)-\nabla_{j} \nabla_{l}\left(h_{i m} h_{n k} g^{m n}\right) \\
& \quad-R_{i j k s}\left(R_{t l}-h_{t m} h_{n l} g^{m n}\right) g^{s t}-R_{i j s l}\left(R_{t k}-h_{t m} h_{n k} g^{m n}\right) g^{s t}
\end{aligned}
$$

and the identity

$$
\begin{aligned}
\triangle R_{i j k l}=-2\left(B_{i j k l}-B_{i j l k}-B_{i l j k}+B_{i k j l}\right)+\nabla_{i} \nabla_{k} R_{j l} & -\nabla_{i} \nabla_{l} R_{j k}-\nabla_{j} \nabla_{k} R_{i l}+\nabla_{j} \nabla_{l} R_{i k} \\
& +R_{m j k l} R_{n i} g^{m n}+R_{i m k l} R_{n j} g^{m n},
\end{aligned}
$$

where $B_{i j k l}=R_{m i j s} R_{n k l t} g^{m n} g^{s t}$. Then we obtain

$$
\begin{aligned}
\left(\partial_{t}-\triangle\right) R_{i j k l} & -2\left(B_{i j k l}-B_{i j l k}-B_{i l j k}+B_{i k j l}\right) \\
= & -R_{i j k s}\left(R_{t l}-h_{t m} h_{n l} g^{m n}\right) g^{s t}-R_{i j s l}\left(R_{t k}-h_{t m} h_{n k} g^{m n}\right) g^{s t} \\
& -R_{s j k l}\left(R_{t i}-h_{t m} h_{n i} g^{m n}\right) g^{s t}-R_{i s k l}\left(R_{t j}-h_{t m} h_{n j} g^{m n}\right) g^{s t} \\
& -R_{s j k l} h_{t m} h_{n i} g^{m n} g^{s t}-R_{i s k l} h_{t m} h_{n j} g^{m n} g^{s t} \\
& -\nabla_{i} \nabla_{k}\left(h_{j m} h_{n l} g^{m n}\right)+\nabla_{i} \nabla_{l}\left(h_{j m} h_{n k} g^{m n}\right) \\
& +\nabla_{j} \nabla_{k}\left(h_{i m} h_{n l} g^{m n}\right)-\nabla_{j} \nabla_{l}\left(h_{i m} h_{n k} g^{m n}\right) .
\end{aligned}
$$


To simplify the evolution equations, we will use a moving frame trick. Let us pick an abstract vector bundle $V$ over $M$ isomorphic to the tangent bundle $T M$. Choose an orthonormal frame $F_{a}=F_{a}^{i} \partial / \partial x^{i}$ for $a=1, \ldots, n$ of $V$ at $t=0$; then evolve $F_{i}^{a}$ by the equation

$$
\partial_{t} F_{a}^{i}=g^{i j}\left(R_{j k}-h_{j m} h_{n k} g^{m n}\right) F_{a}^{k} .
$$

Then the frame $F=\left\{F_{1}, \ldots, F_{a}, \ldots, F_{n}\right\}$ will remain orthonormal for all time. We will use indices $a, b, \ldots$ on a tensor to denote its components in the evolving orthonormal frame. In this frame we have

$$
\begin{aligned}
\left(\partial_{t}-\triangle\right) R_{a b c d}-2\left(B_{a b c d}\right. & \left.-B_{a b d c}-B_{a d c b}+B_{a c b d}\right) \\
=- & R_{s b c d} h_{t m} h_{n a} g^{m n} g^{s t}-R_{a s c d} h_{t m} h_{n b} g^{m n} g^{s t} \\
& \quad-\nabla_{a} \nabla_{c}\left(h_{b m} h_{n d} g^{m n}\right)+\nabla_{a} \nabla_{d}\left(h_{b m} h_{n c} g^{m n}\right) \\
& +\nabla_{b} \nabla_{c}\left(h_{a m} h_{n d} g^{m n}\right)-\nabla_{b} \nabla_{d}\left(h_{a m} h_{n c} g^{m n}\right)
\end{aligned}
$$

and

$$
\left(\partial_{t}-\triangle\right) h_{a b}=-|A|^{2} h_{a b} .
$$

By calculations, we have

$$
\begin{aligned}
\left(\partial_{t}-\triangle\right) & \left(R_{a b c d}-\left(h_{a d} h_{b c}-h_{a c} h_{b d}\right)\right) \\
=2 & \left(B_{a b c d}-B_{a b d c}-B_{a d c b}+B_{a c b d}\right) \\
& \quad-R_{s b c d} h_{t m} h_{n a} g^{m n} g^{s t}-R_{a s c d} h_{t m} h_{n b} g^{m n} g^{s t} \\
& \quad-\nabla_{a} \nabla_{c}\left(h_{b m} h_{n d} g^{m n}\right)+\nabla_{a} \nabla_{d}\left(h_{b m} h_{n c} g^{m n}\right) \\
& +\nabla_{b} \nabla_{c}\left(h_{a m} h_{n d} g^{m n}\right)-\nabla_{b} \nabla_{d}\left(h_{a m} h_{n c} g^{m n}\right) \\
+ & 2|A|^{2}\left(h_{a d} h_{b c}-h_{a c} h_{b d}\right)+2\left(\nabla_{m} h_{a d} \nabla_{n} h_{b c}-\nabla_{m} h_{a c} \nabla_{n} h_{b d}\right) g^{m n} .
\end{aligned}
$$

Then we want to replace $B_{a b c d}$ by

$$
\tilde{B}_{a b c d}=\left(R_{m a b s}-\left(h_{m s} h_{a b}-h_{m b} h_{a s}\right)\right)\left(R_{m c d s}-\left(h_{m s} h_{c d}-h_{m d} h_{c s}\right)\right) g^{m n} g^{s t}
$$

and replace terms including $\nabla h$ and $\nabla \nabla h$ by $C$ and $\nabla C$, respectively. That is,

$$
\begin{aligned}
B_{a b c d}- & B_{a b d c}-B_{a d c b}+B_{a c b d} \\
= & \tilde{B}_{a b c d}-\tilde{B}_{a b d c}-\tilde{B}_{a d c b}+\tilde{B}_{a c b d} \\
& -R_{m a b s} h_{d n} h_{t c} g^{m n} g^{s t}-R_{m c d s} h_{b n} h_{t a} g^{m n} g^{s t} \\
& +R_{m a b s} h_{c n} h_{t d} g^{m n} g^{s t}+R_{m d c s} h_{b n} h_{t a} g^{m n} g^{s t} \\
& -R_{m a d s} h_{n t} h_{c b} g^{m n} g^{s t}+R_{m a d s} h_{c n} h_{t b} g^{m n} g^{s t} \\
& -R_{m b c s} h_{n t} h_{a d} g^{m n} g^{s t}+R_{m b c s} h_{d n} h_{t a} g^{m n} g^{s t}
\end{aligned}
$$




$$
\begin{aligned}
& +R_{\text {macs }} h_{n t} h_{d b} g^{m n} g^{s t}-R_{\text {macs }} h_{d n} h_{t b} g^{m n} g^{s t} \\
& +R_{m b d s} h_{n t} h_{a c} g^{m n} g^{s t}-R_{m b d s} h_{c n} h_{t a} g^{m n} g^{s t} \\
& -h_{a m} h_{b s} h_{c n} h_{d t} g^{m n} g^{s t}+h_{a m} h_{b s} h_{d n} h_{c t} g^{m n} g^{s t} \\
& +h_{a d} h_{b c}|A|^{2}-h_{a m} h_{d s} h_{n t} h_{b c} g^{m n} g^{s t}-h_{b m} h_{c s} h_{n t} h_{a d} g^{m n} g^{s t} \\
& -h_{a c} h_{b d}|A|^{2}+h_{a m} h_{c s} h_{n t} h_{b d} g^{m n} g^{s t}+h_{b m} h_{d s} h_{n t} h_{a c} g^{m n} g^{s t}
\end{aligned}
$$

and

$$
\begin{aligned}
-\nabla_{a} & \nabla_{c}\left(h_{b m} h_{n d} g^{m n}\right)+\nabla_{a} \nabla_{d}\left(h_{b m} h_{n c} g^{m n}\right)+\nabla_{b} \nabla_{c}\left(h_{a m} h_{n d} g^{m n}\right) \\
-\nabla_{b} & \nabla_{d}\left(h_{a m} h_{n c} g^{m n}\right)+2\left(\nabla_{m} h_{a d} \nabla_{n} h_{b c} g^{m n}-\nabla_{m} h_{a c} \nabla_{n} h_{b d} g^{m n}\right) \\
= & -\nabla_{c}\left(\nabla_{a} h_{b m}-\nabla_{b} h_{a m}\right) h_{n d} g^{m n}-\nabla_{a}\left(\nabla_{c} h_{d m}-\nabla_{d} h_{c m}\right) h_{n b} g^{m n} \\
& +\nabla_{d}\left(\nabla_{a} h_{b m}-\nabla_{b} h_{a m}\right) h_{n c} g^{m n}-\nabla_{b}\left(\nabla_{c} h_{d m}-\nabla_{d} h_{c m}\right) h_{n a} g^{m n} \\
& -\left(\nabla_{a} h_{b m}-\nabla_{b} h_{a m}\right)\left(\nabla_{c} h_{d n}-\nabla_{d} h_{c n}\right) g^{m n} \\
& -\left(\nabla_{a} h_{d m}-\nabla_{m} h_{a d}\right) \nabla_{c} h_{b n} g^{m n}-\left(\nabla_{d} h_{a m}-\nabla_{m} h_{a d}\right) \nabla_{b} h_{c n} g^{m n} \\
& +\left(\nabla_{a} h_{c m}-\nabla_{m} h_{a c}\right) \nabla_{d} h_{b n} g^{m n}+\left(\nabla_{c} h_{a m}-\nabla_{m} h_{a c}\right) \nabla_{b} h_{d n} g^{m n} \\
& +\left(\nabla_{m} h_{b c}-\nabla_{c} h_{m b}\right) \nabla_{n} h_{a d} g^{m n}+\left(\nabla_{m} h_{b c}-\nabla_{b} h_{m c}\right) \nabla_{n} h_{a d} g^{m n} \\
& -\left(\nabla_{m} h_{b d}-\nabla_{d} h_{m b}\right) \nabla_{n} h_{a c} g^{m n}-\left(\nabla_{m} h_{b d}-\nabla_{b} h_{m d}\right) \nabla_{n} h_{a c} g^{m n} \\
& -R_{a c b m} h_{n s} h_{t d} g^{m n} g^{s t}-R_{a c m s} h_{n d} h_{t b} g^{m n} g^{s t}+R_{b c a m} h_{n s} h_{t d} g^{m n} g^{s t} \\
& +R_{b c m s} h_{n d} h_{t a} g^{m n} g^{s t}+R_{a d b m} h_{n s} h_{t c} g^{m n} g^{s t}+R_{a d m s} h_{n c} h_{t b} g^{m n} g^{s t} \\
& -R_{b d a m} h_{n s} h_{t c} g^{m n} g^{s t}-R_{b d m s} h_{n c} h_{t a} g^{m n} g^{s t} .
\end{aligned}
$$

Let us denote the curvature tensor by $R m$ and denote any tensor product of tensors $S$ and $T$ by $S * T$ when we do not need the precise expression. If we replace terms including $R m * h * h$ by terms $G * h * h$ and if we use (3-4), (3-5) and (3-6), then some calculations gives

$$
\left(\partial_{t}-\triangle\right) G=G * G+G * h * h+\nabla C * h+C * \nabla h+C * C,
$$

where $G_{i j k l}=R_{i j k l}-\left(h_{i l} h_{j k}-h_{i k} h_{j l}\right)$ and $C_{i j k}=\nabla_{i} h_{j k}-\nabla_{j} h_{i k}$. Since

$$
\begin{aligned}
\partial_{t} \nabla_{i} h_{j k}=\nabla_{i}\left(\partial_{t} h_{j k}\right)-\left(\partial_{t} \Gamma_{i j}^{l}\right) h_{l k}-\left(\partial_{t} \Gamma_{i k}^{l}\right) h_{l j} \\
=\nabla_{i}\left(\triangle h_{j k}-R_{j m} h_{n k} g^{m n}-R_{k m} h_{n j} g^{m n}+2 h_{j m} h_{n s} h_{t k} g^{m n} g^{s t}-|A|^{2} h_{j k}\right) \\
\quad-\left(\partial_{t} \Gamma_{i j}^{l}\right) h_{l k}+\nabla_{i} R_{k m} h_{n j} g^{m n}+\nabla_{k} R_{i m} h_{n j} g^{m n}-\nabla_{m} R_{i k} h_{n j} g^{m n} \\
\quad-\nabla_{i} h_{k m} h_{n s} h_{t j} g^{m n} g^{s t}-\nabla_{i} h_{m s} h_{n k} h_{t j} g^{m n} g^{s t}-\nabla_{k} h_{i m} h_{n s} h_{t j} g^{m n} g^{s t} \\
\quad-\nabla_{k} h_{m s} h_{n i} h_{t j} g^{m n} g^{s t}+\nabla_{m} h_{i s} h_{n j} h_{t k} g^{m n} g^{s t}+\nabla_{m} h_{k s} h_{n j} h_{t i} g^{m n} g^{s t}
\end{aligned}
$$


and

$$
\begin{aligned}
\Delta\left(\nabla_{i} h_{j k}\right)= & g^{m n} \nabla_{m} \nabla_{n}\left(\nabla_{i} h_{j k}\right) \\
= & \nabla_{i}\left(\triangle h_{j k}\right)+R_{i m} \nabla_{n} h_{j k} g^{m n}+2\left(R_{m i j s} \nabla_{n} h_{t k}+R_{m i k s} \nabla_{n} h_{t j}\right) g^{m n} g^{s t} \\
& +\nabla_{j} R_{i m} h_{n k} g^{m n}-\nabla_{m} R_{i j} h_{n k} g^{m n}+\nabla_{k} R_{i m} h_{n j} g^{m n}-\nabla_{m} R_{i k} h_{n j} g^{m n},
\end{aligned}
$$

we get

$$
\begin{aligned}
\left(\partial_{t}-\triangle\right) \nabla_{i} & h_{j k}+\left(\partial_{t} \Gamma_{i j}^{l}\right) h_{l k} \\
= & -R_{j m} \nabla_{i} h_{n k} g^{m n}-R_{k m} \nabla_{i} h_{n j} g^{m n}-R_{i m} \nabla_{n} h_{j k} g^{m n} \\
& +\nabla_{i}\left(2 h_{j m} h_{n s} h_{t k} g^{m n} g^{s t}-|A|^{2} h_{j k}\right) \\
& -\nabla_{i} R_{j m} h_{n k} g^{m n}-\nabla_{j} R_{i m} h_{n k} g^{m n} \\
& -2\left(R_{m i j s} \nabla_{n} h_{t k}+R_{m i k s} \nabla_{n} h_{t j}\right) g^{m n} g^{s t} \\
& -\nabla_{i} h_{k m} h_{n s} h_{t j} g^{m n} g^{s t}-\nabla_{i} h_{m s} h_{n k} h_{t j} g^{m n} g^{s t} \\
& -\nabla_{k} h_{i m} h_{n s} h_{t j} g^{m n} g^{s t}-\nabla_{k} h_{m s} h_{n i} h_{t j} g^{m n} g^{s t} \\
& +\nabla_{m} h_{i s} h_{t k} h_{n j} g^{m n} g^{s t}+\nabla_{m} h_{k s} h_{t i} h_{n j} g^{m n} g^{s t} .
\end{aligned}
$$

Then in the moving frame we obtain

$$
\begin{aligned}
\left(\partial_{t}-\right. & \triangle) \nabla_{a} h_{b c}+|A|^{2} \nabla_{a} h_{b c}+\left(\partial_{t} \Gamma_{i j}^{l}\right) h_{l k} F_{a}^{i} F_{b}^{j} F_{c}^{k} \\
= & -\nabla_{a} h_{c m} h_{n s} h_{t b} g^{m n} g^{s t}-\nabla_{a} h_{m b} h_{n s} h_{t c} g^{m n} g^{s t}-\nabla_{m} h_{b c} h_{n s} h_{t a} g^{m n} g^{s t} \\
& +2 \nabla_{a} h_{b m} h_{n s} h_{t k c} g^{m n} g^{s t}+2 \nabla_{a} h_{c m} h_{n s} h_{t b} g^{m n} g^{s t} \\
& +2 \nabla_{a} h_{m s} h_{n b} h_{t c} g^{m n} g^{s t}-2 \nabla_{a} h_{m s} h_{n t} h_{b c} g^{m n} g^{s t} \\
& -\nabla_{a} h_{c m} h_{n s} h_{t b} g^{m n} g^{s t}-\nabla_{a} h_{m s} h_{n b} h_{t c} g^{m n} g^{s t}-\nabla_{c} h_{a m} h_{n s} h_{t b} g^{m n} g^{s t} \\
& +\nabla_{m} h_{a s} h_{n b} h_{t c} g^{m n} g^{s t}+\nabla_{m} h_{c s} h_{n b} h_{t a} g^{m n} g^{s t} \\
& -2 R_{m a b s} \nabla_{n} h_{t c} g^{m n} g^{s t}-2 R_{m a c s} \nabla_{n} h_{t b} g^{m n} g^{s t} .
\end{aligned}
$$

Then we replace terms including $\nabla h$ by $C$ and terms including $R m$ by $G$. Finally, we have

$$
\left(\partial_{t}-\triangle\right) C=-|A|^{2} C+C * h * h+C * R m+G * \nabla h .
$$

Combing (3-7) and (3-10), we obtain

$$
\begin{aligned}
\left(\partial_{t}-\triangle\right)\left(|G|^{2}+|C|^{2}\right) & \\
\leq & C_{1}\left(|G|^{2}+|C|^{2}\right)-2|\nabla G|^{2}-2|\nabla C|^{2} \\
& +\langle G, G * G+G * h * h+\nabla C * h+C * \nabla h+C * C\rangle \\
& +\left\langle C,-|A|^{2} C+C * h * h+C * R m+G * \nabla h\right\rangle \\
\leq & C_{2}\left(|G|^{2}+|C|^{2}\right),
\end{aligned}
$$


where we use the Cauchy-Schwarz inequality, and for $0 \leq t<\delta$ we have bounded $|R m|,|A|$ and $|\nabla h|$. Thus, by the standard maximum principle

$$
\frac{d}{d t}\left(|G|^{2}+|C|^{2}\right)_{\max } \leq C_{2}\left(|G|^{2}+|C|^{2}\right)_{\max },
$$

we get

$$
\left(|G|^{2}+|C|^{2}\right)_{\max }(t) \leq e^{C_{2} t}\left(|G|^{2}+|C|^{2}\right)_{\max }(0) .
$$

Since $\left(|G|^{2}+|C|^{2}\right)_{\max }(0)=0$, the Gauss-Codazzi equations are preserved as long as the solution exists.

In the following we will still call $h_{i j}(x, t)$ the second fundamental form and its trace $H$ the mean curvature.

\section{Evolution of the metric and curvature}

Using the Gauss-Codazzi equations, we rewrite our evolution equations:

\section{Proposition 4.1.}

$$
\begin{aligned}
\partial_{t} g_{i j} & =2 H h_{i j} . \\
\left(\partial_{t}-\triangle\right) h_{i j} & =2 H h_{i m} h_{n j} g^{m n}-|A|^{2} h_{i j} . \\
\left(\partial_{t}-\triangle\right) H & =-H|A|^{2} . \\
\left(\partial_{t}-\triangle\right)|A|^{2} & =-2|\nabla A|^{2}-2|A|^{4} .
\end{aligned}
$$

Since $h_{i j}$ is positive at $t=0$ and $M$ is compact, there are some $\varepsilon>0$ and $\beta>0$ such that $\beta H g_{i j} \geq h_{i j} \geq \varepsilon H g_{i j}$ holds on $M$ at $t=0$. We want to show that inequality remains true as long as the solution of our evolution equations (1-1) exists. For this purpose we need the following maximum principle for tensors on manifolds, which is proved in [Hamilton 1982].

On a compact manifold $M$, let $u^{k}$ be a vector field and $M_{i j}$ and $N_{i j}$ be symmetric tensors, all of which may depend on time $t$. Assume that $N_{i j}=p\left(M_{i j}, g_{i j}\right)$ is a polynomial in $M_{i j}$ formed by contracting products of $M_{i j}$ with itself using the metric. Suppose this polynomial satisfies the condition $N_{i j} X^{i} X^{j} \geq 0$ for any nulleigenvector $X$ of $M_{i j}$.

Theorem 4.2 [Hamilton 1986]. Suppose that the evolution equation

$$
\partial_{t} M_{i j}=\triangle M_{i j}+u^{k} \nabla_{k} M_{i j}+N_{i j}
$$

holds on $0 \leq t<T$, where $N_{i j}=p\left(M_{i j}, g_{i j}\right)$ satisfies the null-eigenvector condition above. If $M_{i j} \geq 0$ at $t=0$, then it remains so on $0 \leq t<T$.

Proposition 4.3. If $\varepsilon H g_{i j} \leq h_{i j} \leq \beta H g_{i j}$ and $H>0$ at $t=0$, then these relations continue to hold as long as the solution of (1-1) exists. 
Proof. First, by using maximum principle on the equation $\left(\partial_{t}-\triangle\right) H=-H|A|^{2}$, we know $H>0$ as long as the solution of (1-1) exists. Then we consider

$$
\begin{aligned}
M_{i j} & =h_{i j}-\varepsilon H g_{i j}, \\
\partial_{t} M_{i j} & =\partial_{t} h_{i j}-\varepsilon \partial_{t} H g_{i j}-\varepsilon H \partial_{t} g_{i j} \\
& =\triangle h_{i j}+2 H h_{i m} h_{n j} g^{m n}-|A|^{2} h_{i j}-\varepsilon\left(\triangle H-|A|^{2} H\right) g_{i j}-\varepsilon H\left(2 H h_{i j}\right) \\
& =\triangle M_{i j}+2 H h_{i m} h_{n j} g^{m n}-|A|^{2}\left(h_{i j}-\varepsilon H g_{i j}\right)-2 \varepsilon H^{2} h_{i j} .
\end{aligned}
$$

For any null vector $v^{i}$ of $M_{i j}$, we have

$$
\begin{aligned}
\left(2 H h_{i m} h_{n j} g^{m n}-|A|^{2}\left(h_{i j}-\varepsilon\right.\right. & \left.\left.H g_{i j}\right)-2 \varepsilon H^{2} h_{i j}\right) v^{j} \\
= & 2 H h_{i m} g^{m n}\left(\varepsilon H v_{n}\right)-2 \varepsilon H^{2}\left(\varepsilon H v_{i}\right) \\
= & 2 H\left(\varepsilon H v_{i}\right) \varepsilon H-2 \varepsilon H^{2}\left(\varepsilon H v_{i}\right)=0 .
\end{aligned}
$$

Thus, $\varepsilon H g_{i j} \leq h_{i j}$ follows from Theorem 4.2. Then $h_{i j} \leq \beta H g_{i j}$ follows in the same way.

Finally, we state the higher derivative estimate.

Proposition 4.4. There exist constants $C_{m}$ for $m=1,2, \ldots$ such that if the second fundamental form of a complete solution to our evolution equation is bounded by $|A| \leq M$ up to time $t$ with $0<t \leq 1 / M$, then the covariant derivative of the second fundamental form is bounded by

$$
|\nabla A| \leq C_{1} M / \sqrt{t}
$$

and the $m$-th covariant derivative of the second fundamental form is bounded by

$$
\left|\nabla^{m} A\right| \leq C_{m} M / t^{m / 2} .
$$

Here the norms are taken with respect to the evolving metric.

Proof. By direct calculation, for any $m$ we have an equation

$$
\left(\partial_{t}-\triangle\right)\left|\nabla^{m} A\right|^{2}=-2\left|\nabla^{m+1} A\right|^{2}+\sum_{i+j+k=m} \nabla^{i} A * \nabla^{j} A * \nabla^{k} A * \nabla^{m} A .
$$

So we can follow the same way using a somewhat standard Bernstein estimate in partial differential equations to get our theorem; see [Shi 1989] for the argument in the case of Ricci flow.

\section{Monotonicity formula and long-time behaviors}

First, by the positivity of $h_{i j}$ we have $H^{2} / n \leq|A|^{2}<H^{2}$. Then from (4-1c) we get

$$
-H^{3}<\left(\partial_{t}-\triangle\right) H \leq-H^{3} / n
$$


Thus by maximum principle we obtain

$$
\frac{1}{\sqrt{2 t+1 / H_{\min }^{2}(0)}}<H(t) \leq \frac{1}{\sqrt{2 t / n+1 / H_{\max }^{2}(0)}} .
$$

With applying maximum principle on (4-1d), we have

$$
|A|^{2}(t) \leq \frac{1}{2 t+1 /|A|_{\max }^{2}(0)}
$$

Since

$$
\frac{1}{2 n t+n / H_{\min }^{2}(0)}<H^{2}(t) / n \leq|A|^{2}(t)
$$

we get

$$
\frac{1}{2 n t+n / H_{\min }^{2}(0)}<|A|^{2}(t) \leq \frac{1}{2 t+1 /|A|_{\max }^{2}(0)} .
$$

In particular, (5-2) implies $|A| \rightarrow 0$ as $t \rightarrow+\infty$. Combining this with our derivatives estimate, Proposition 4.4, we conclude the solution of (1-1) exists for all time.

We need the monotonicity formula below to understand the long-time behavior of the solution to (1-1).

Proposition 5.1. If $\left(g_{i j}(t), h_{i j}(t)\right)$ is the solution of (1-1), then we have the formula

$$
\frac{\partial}{\partial t} \int_{M} H^{n} d \mu_{t}=-n(n-1) \int_{M} \frac{|\nabla H|^{2}}{H^{2}} H^{n} d \mu_{t}-n \int_{M}\left|h_{i j}-\frac{1}{n} H g_{i j}\right|^{2} H^{n} d \mu_{t} .
$$

Proof. It follows from the evolution equations of Proposition 4.1 and direct calculation.

From Proposition 5.1, we know

$$
0<\int_{M} H^{n} d \mu_{t}<C \quad \text { for all } t \in[0,+\infty) .
$$

This implies

$$
\int_{0}^{\infty} \int_{M}\left(\frac{|\nabla H|^{2}}{H^{2}}+\left|h_{i j}-\frac{1}{n} H g_{i j}\right|^{2}\right) H^{n} d \mu_{t} d t<\infty .
$$

In particular, there is a sequence $t_{k} \rightarrow+\infty$ such that

$$
\begin{aligned}
t_{k} \int_{M} \frac{|\nabla H|^{2}}{H^{2}} H^{n} d \mu_{t_{k}} \rightarrow 0 \quad \text { as } k \rightarrow \infty, \\
t_{k} \int_{M}\left|h_{i j}-\frac{1}{n} H g_{i j}\right|^{2} H^{n} d \mu_{t_{k}} \rightarrow 0 \quad \text { as } k \rightarrow \infty .
\end{aligned}
$$


Let $\epsilon_{k}=1 /|A|_{\max }\left(t_{k}\right)$. We parabolically scale the solution and shift the time $t_{k}$ to the origin 0 by letting

$$
\begin{aligned}
& \tilde{g}_{i j}^{k}(\cdot, \tilde{t})=\epsilon_{k}^{-2} g_{i j}\left(\cdot, t_{k}+\epsilon_{k}^{2} \tilde{t}\right), \\
& \tilde{h}_{i j}^{k}(\cdot, \tilde{t})=\epsilon_{k}^{-1} h_{i j}\left(\cdot, t_{k}+\epsilon_{k}^{2} \tilde{t}\right), \quad \text { where } \tilde{t} \in\left[-t_{k} / \epsilon_{k}^{2},+\infty\right) .
\end{aligned}
$$

We can check that $\left(\tilde{g}_{i j}^{k}(\cdot, \tilde{t}), \tilde{h}_{i j}^{k}(\cdot, \tilde{t})\right)$ is still a solution to $(1-1)$. From

$$
\left|\tilde{A}^{k}(\cdot, \tilde{t})\right|^{2}=\left|A\left(\cdot, t_{k}+\epsilon_{k}^{2} \tilde{t}\right)\right|^{2} /|A|_{\max }^{2}\left(t_{k}\right)
$$

and (5-2), it follows that

$$
1 / C_{1}<\left|\tilde{A}^{k}(\cdot, \tilde{t})\right|^{2}<C_{1} \quad \text { for } \tilde{t} \in\left[-t_{k} / 2 \epsilon_{k}^{2}, 0\right],
$$

where the constant $C_{1}$ is independent of $k$.

By our derivatives estimate, Proposition 4.4, the uniform bound of the second fundamental form $\left|\tilde{A}^{k}(\cdot, \tilde{t})\right|$ implies the uniform bound on all the derivatives of the second fundamental form at $\tilde{t}=0$ for all $k$. By Gauss's equation, we have uniform bounds of the curvature and all its derivatives at $\tilde{t}=0$ for all $k$.

By (5-3) we know that $\int_{M}\left(\tilde{H}^{k}(\cdot, 0)\right)^{n} d \tilde{\mu}_{0}<C_{2}$. Combining this with (5-1), we find

$$
\operatorname{Vol}\left(M, \tilde{g}_{i j}^{k}(\cdot, 0)\right)<C_{3} .
$$

On the other hand, by Proposition 4.3, (5-2), and the Gauss equation, we have

$$
0>-1 / C_{4} \geq \operatorname{Sec}\left(M, \tilde{g}_{i j}^{k}(\cdot, 0)\right) \geq-1,
$$

where Sec means sectional curvature.

Theorem 5.2 [Gromov 1978]. Let $M$ be an n-dimensional closed Riemannian manifold of negative curvature and suppose $\operatorname{Sec}(M) \geq-1$. Then

$$
\operatorname{Vol}(M) \geq \begin{cases}C(1+d(M)) & \text { if } n \geq 8, \\ C\left(1+d^{1 / 3}(M)\right) & \text { if } n=4,5,6,7,\end{cases}
$$

where we denote by $\operatorname{Vol}(M)$ and $d(M)$ the volume and diameter of $M$, and the constant $C>0$ depends only on $n$.

Combining (5-8) and (5-7) and this theorem of Gromov, we have

$$
\operatorname{diam}\left(M, \tilde{g}_{i j}^{k}(\cdot, 0)\right) \leq C_{5} \quad \text { and } \quad \operatorname{Vol}\left(M, \tilde{g}_{i j}^{k}(\cdot, 0)\right) \geq 1 / C_{5} .
$$

Now we know $\left(M, \tilde{g}_{i j}^{k}(\cdot, 0), \tilde{h}_{i j}^{k}(\cdot, 0)\right)$ is a sequence of Riemannian manifolds that have uniformly bounded sectional curvature, uniform upper bound on their diameters, and uniform lower bound on their volumes. Using Cheeger's lemma in [Cheeger and Ebin 1975], we have the uniform lower bound of their injective radii with respect to $\tilde{g}_{i j}^{k}(\cdot, 0)$ for $n \geq 4$. Then we can apply the argument used 
to prove Hamilton's compactness theorem of [1995] to extract a convergent subsequence $\left(M, \tilde{g}_{i j}^{k_{l}}(\cdot, 0), \tilde{h}_{i j}^{k_{l}}(\cdot, 0)\right)$ from $\left(M, \tilde{g}_{i j}^{k}(\cdot, 0), \tilde{h}_{i j}^{k}(\cdot, 0)\right)$. More precisely, there exists a triple $\left(M_{\infty}, \tilde{g}_{i j}^{\infty}(\cdot, 0), \tilde{h}_{i j}^{\infty}(\cdot, 0)\right)$ and a sequence of diffeomorphisms $f_{l}: M_{\infty} \rightarrow M_{l}$. Notice that $M_{\infty}$ is diffeomorphic to $M$, since we have uniform diameter bound. Also the pull-back metrics $\left(f_{l}\right)^{*} \tilde{g}_{i j}^{k_{l}}(\cdot, 0)$ and second fundamental forms $\left(f_{l}\right)^{*} \tilde{h}_{i j}^{k_{l}}(\cdot, 0)$ converge in the $C^{\infty}$ topology to $\left(\tilde{g}_{i j}^{\infty}(\cdot, 0), \tilde{h}_{i j}^{\infty}(\cdot, 0)\right)$.

From (5-4) and (5-5) we obtain

$$
\begin{array}{r}
t_{k_{l}} \epsilon_{k_{l}}^{-2} \int_{M} \frac{\left|\tilde{\nabla} \tilde{H}^{k_{l}}\right|^{2}(0)}{\left(\tilde{H}^{k_{l}}\right)^{2}(0)}\left(\tilde{H}^{k_{l}}\right)^{n}(0) d \tilde{\mu}_{t_{k_{l}}} \rightarrow 0 \quad \text { as } l \rightarrow \infty, \\
t_{k_{l}} \epsilon_{k_{l}}^{-2} \int_{M}\left|\tilde{h}_{i j}^{k_{l}}-\frac{1}{n} \tilde{H}^{k_{l}} \tilde{g}_{i j}^{k_{l}}\right|^{2}(0)\left(\tilde{H}^{k_{l}}\right)^{n}(0) d \tilde{\mu}_{t_{k_{l}}} \rightarrow 0 \quad \text { as } l \rightarrow \infty .
\end{array}
$$

Here the norm is taken with respect to $\tilde{g}_{i j}^{k_{l}}(0)$. Noting that $t_{k_{l}} \epsilon_{k_{l}}^{-2}$ and $\left|\tilde{H}^{k_{l}}(0)\right|$ and $\operatorname{Vol}\left(M, \tilde{g}_{i j}^{k_{l}}(\cdot, 0)\right)$ have uniform lower bound, we have

$$
\begin{aligned}
\left|\tilde{\nabla} \tilde{H}^{k_{l}}\right|(0) \rightarrow 0 & \text { as } l \rightarrow \infty, \\
\left|\tilde{h}_{i j}^{k_{l}}-\frac{1}{n} \tilde{H}^{k_{l}} \tilde{g}_{i j}^{k_{l}}\right|(0) \rightarrow 0 & \text { as } l \rightarrow \infty .
\end{aligned}
$$

Therefore, by Gauss's equation, we know $\operatorname{Sec}\left(M_{\infty}, \tilde{g}_{i j}^{\infty}(\cdot, 0), \tilde{h}_{i j}^{\infty}(\cdot, 0)\right)$ is a constant equal to $-1 / n$.

\section{Acknowledgments}

I am grateful to my advisor Professor B. L. Chen for his guidance.

\section{References}

[Cheeger and Ebin 1975] J. Cheeger and D. G. Ebin, Comparison theorems in Riemannian geometry, North-Holland Mathematical Library 9, North-Holland, Amsterdam, 1975. MR 56 \#16538 Zbl 0309.53035

[Ecker 1997] K. Ecker, "Interior estimates and longtime solutions for mean curvature flow of noncompact spacelike hypersurfaces in Minkowski space", J. Differential Geom. 46:3 (1997), 481-498. MR 98j:58034 Zbl 0909.53045

[Ecker and Huisken 1991] K. Ecker and G. Huisken, "Parabolic methods for the construction of spacelike slices of prescribed mean curvature in cosmological spacetimes", Comm. Math. Phys. 135:3 (1991), 595-613. MR 92g:53058 Zbl 0721.53055

[Gromov 1978] M. Gromov, "Manifolds of negative curvature", J. Differential Geom. 13:2 (1978), 223-230. MR 80h:53040 Zbl 0433.53028

[Gromov and Thurston 1987] M. Gromov and W. Thurston, "Pinching constants for hyperbolic manifolds", Invent. Math. 89:1 (1987), 1-12. MR 88e:53058 Zbl 0646.53037

[Hamilton 1982] R. S. Hamilton, "Three-manifolds with positive Ricci curvature", J. Differential Geom. 17:2 (1982), 255-306. MR 84a:53050 Zbl 0504.53034 
[Hamilton 1986] R. S. Hamilton, "Four-manifolds with positive curvature operator", J. Differential Geom. 24:2 (1986), 153-179. MR 87m:53055 Zbl 0628.53042

[Hamilton 1995] R. S. Hamilton, "The formation of singularities in the Ricci flow", pp. 7-136 in Surveys in differential geometry, II (Cambridge, MA, 1993), edited by S.-T. Yau, Int. Press, Cambridge, MA, 1995. MR 97e:53075 Zbl 0867.53030

[Huisken 1984] G. Huisken, "Flow by mean curvature of convex surfaces into spheres", J. Differential Geom. 20:1 (1984), 237-266. MR 86j:53097 Zbl 0556.53001

[Shi 1989] W.-X. Shi, "Deforming the metric on complete Riemannian manifolds", J. Differential Geom. 30:1 (1989), 223-301. MR 90i:58202 Zbl 0676.53044

Received February 25, 2009.

KUN ZHANG

SCHOol of Mathematics AND COMPUTATIONAL SCIENCE

SUN YAT-SEN UNIVERSITY

No. 135 Xingang RoAd West

GUANGZHOU 510275

CHINA

zhangkun@mail2.sysu.edu.cn 